Service social

\title{
Le groupe en contexte de réadaptation. Évaluation d'une intervention auprès des personnes atteintes de maladie pulmonaire obstructive chronique
}

\section{Lyse Montminy et Jean-Claude Bellavance}

Volume 45, numéro 3, 1996

Santé

URI : https://id.erudit.org/iderudit/706743ar

DOI : https://doi.org/10.7202/706743ar

Aller au sommaire du numéro

Éditeur(s)

École de service social de l'Université Laval

ISSN

1708-1734 (numérique)

Découvrir la revue

Citer cet article

Montminy, L. \& Bellavance, J.-C. (1996). Le groupe en contexte de réadaptation. Évaluation d'une intervention auprès des personnes atteintes de maladie pulmonaire obstructive chronique. Service social, 45(3), 153-174. https://doi.org/10.7202/706743ar
Résumé de l'article

L'objectif de cette recherche est d'aider la personne atteinte d'une maladie pulmonaire obstructive chronique à fonctionner de façon optimale à l'intérieur des limites imposées par sa maladie. Pour y arriver, nous avons formé des groupes de soutien et d'éducation en vue d'améliorer la qualité de vie des participants. Cet article décrit d'abord l'intervention telle que réalisée et présente les résultats d'analyses qui évaluent les changements de comportements des participants au groupe. Ces changements vont pour la plupart dans le sens d'une amélioration de leur qualité de vie. Les analyses des résultats obtenus auprès des participants tendent aussi à démontrer que l'intervention de groupe favorise le développement de moyens permettant d'apprendre à mieux vivre avec la MPOC et ses effets. Les plus importants apprentissages des participants au groupe sont l'amorce d'une prise de conscience à l'égard de la maladie et de ses impacts et une meilleure disposition à l'action. 


\section{Le groupe en contexte de réadaptation}

Évaluation d'une intervention auprès des personnes atteintes de maladie pulmonaire obstructive chronique

Lyse MONTMINY Étudiante au doctorat en service social Chargée de cours Université Laval Jean-Claude BELLAVANCE Travailleur social, Hôpital Laval

Les maladies pulmonaires obstructives chroniques (MPOC) représentent chez les personnes âgées de 65 ans et plus le sixième problème de santé le plus fréquent (Bracuk, 1993). Ces maladies sont susceptibles de provoquer des difficultés allant d'une limitation intermittente des activités à l'incapacité totale et permanente (Rabinowitz et Florian, 1992 ; Lee, Graydon et Ross, 1991). 
On peut facilement comprendre qu'un tel handicap puisse être à l'origine de difficultés psychosociales et psychologiques majeures (Dudley et al., 1980), non seulement pour le malade, mais aussi pour son entourage (Rabinowitz et Florian, 1992; Gayle, Foxall et Barron, 1993 ; Sexton et Munro, 1985).

Bon nombre d'auteurs (Ries et al., 1995 ; Emery et al., 1991; Celle, 1995 ; Ojanen et al., 1993) ont montré qu'un programme de réadaptation peut diminuer l'essoufflement, l'anxiété et la dépression, améliorer la tolérance à l'effort, faciliter l'accomplissement des activités de la vie quotidienne et, par corollaire, améliorer la qualité de vie. Bien qu'il soit reconnu que le soutien social (Graydon et Ross, 1995), exerce une influence sur la qualité de vie des personnes atteintes de MPOC, peu d'études traitent spécifiquement de l'intervention de groupe comme moyen de favoriser la réadaptation du malade pulmonaire.

Après avoir fait état d'une brève recension des écrits sur les impacts de la maladie, nous présenterons dans cet article l'évaluation d'une intervention de groupe réalisée auprès de personnes atteintes de MPOC et de leurs proches. Dans un premier temps, nous décrirons l'intervention à partir de ses objectifs, de ses appuis théoriques et de son contenu. En second lieu, nous présenterons les résultats de l'intervention eu égard aux changements survenus pendant la période entourant l'intervention ainsi qu'aux contributions des participants à l'amélioration de la qualité de vie des membres du groupe.

\section{LES IMPACTS DE LA MALADIE PULMONAIRE OBSTRUCTIVE CHRONIQUE}

La maladie pulmonaire obstructive chronique (MPOC) est, comme son nom l'indique, chronique, mais elle est aussi évolutive. Elle affecte tout aussi bien les hommes que les femmes. Toutefois, ce sont davantage des hommes dont l'âge se situe autour de 65 ans qui en sont atteints (Bracuk, 1993). Le tabagisme constitue le principal facteur de risque dans l'évolution de cette maladie qui se développera chez environ $10 \%$ à $15 \%$ des grands fumeurs (Bracuk, 1993; Tames, 1991). Parmi les autres facteurs pouvant exercer un effet sur l'apparition de cette maladie, on trouve la pollution atmosphérique, l'exposition professionnelle à des produits toxiques, les infections pulmonaires chroniques ainsi que l’hérédité (Tames, 1991). 
La MPOC représente la sixième cause de décès au Canada chez les hommes et la huitième chez les femmes (Manfreda et Litvin, 1992). Plus de 635000 Canadiens de tout âge souffrent de bronchite et d'emphysème. De ce nombre, quelque 25000 sont hospitalisés chaque année (Bracuk, 1993). La maladie pulmonaire obstructive chronique est définie comme irréversible, ce qui signifie que les dommages qu'elle entraîne sont permanents. Toutefois, il demeure possible par une médication appropriée d'en diminuer les symptômes et d'en retarder la progression.

Progressant lentement et insidieusement, la maladie pulmonaire obstructive chronique se manifeste, entre autres, par une diminution de la tolérance à l'effort et un accroissement de l'essoufflement. À peine perceptible au début, l'essoufflement est de plus en plus manifeste au rythme de la progression de la maladie et il devient invalidant à un stade avancé. Devant la progression constante de l'intolérance à l'effort, le patient se voit contraint de diminuer, et même parfois de cesser, les activités sociales et physiques qui requièrent un effort (Lee et al., 1991). Les activités de la vie quotidienne peuvent même devenir pénibles en raison de l'essoufflement au moindre effort.

Les répercussions de la maladie sont nombreuses. Elles affectent tout aussi bien le malade que ses proches et son réseau social (Lee et al., 1991; Graydon et Ross, 1995). Le malade pulmonaire est sujet à la dépression, à l'anxiété et à la panique (Light et al., 1985; Gayle et al., 1993) La réaction dépressive entraînée par le caractère chronique, évolutif et limitatif de la maladie peut être associée au fait que la personne atteinte éprouve de la difficulté à faire le deuil de son état de santé antérieur. Renoncer à bon nombre d'activités ou de projets exige de la personne de puiser à même ses ressources personnelles afin de surmonter cette épreuve. Face à une telle situation, la présence d'un réseau de soutien facilite (Gayle et al., 1993) le passage au nouveau mode de vie que devra adopter le malade pulmonaire.

Quant aux réactions d'anxiété et parfois même de panique, elles sont souvent associées à l'intolérance à l'effort qui provoque la peur de suffoquer ou de manquer d'air (Lauzon et Adam, 1996). La réponse de la personne à cette situation d'anxiété est d'éviter tout effort physique pouvant conduire à l'essoufflement.

On observe fréquemment chez la personne une baisse de l'estime de soi (Bracuk, 1991), qui se manifeste par un sentiment d'inutilité pouvant s'expliquer entre autres par la diminution graduelle de ses capacités (Lauzon et Adam, 1996) et la 
comparaison qu'elle fait avec d'autres personnes du même âge n'ayant pas d'atteintes pulmonaires.

Pour favoriser chez la personne atteinte de MPOC un fonctionnement maximal à l'intérieur des limites imposées par sa maladie, le département de pneumologie de l'Hôpital Laval a mis sur pied un programme intégré de réadaptation à l'intérieur duquel plusieurs professionnels, dont les travailleurs sociaux, sont engagés. Dans le but de répondre aux problèmes d'ordre psychosocial ainsi qu'aux répercussions de la maladie influant sur la qualité de vie, différents types d'intervention sont offerts, dont un groupe de soutien.

Ce dernier type d'intervention a pour objectif de réunir des personnes vivant le même problème, afin de leur permettre de prendre conscience qu'elles ne sont pas seules dans leur situation. L'intervention de groupe permet aux participants de briser leur isolement en favorisant l'expression du vécu et des émotions. Ce type d'intervention peut aussi contribuer à l'identification de moyens favorisant une meilleure qualité de vie et un pouvoir accru des participants sur leur vie personnelle, familiale et sociale. L'objet de cet article est de présenter les résultats d'une intervention de groupe menée à l'intérieur d'un programme de réadaptation dont l'objectif principal était d'améliorer la qualité de vie des personnes atteintes de MPOC.

\section{L'INTERVENTION: OBJECTIFS, APPUIS THÉORIQUES ET DESCRIPTION}

\section{Les objectifs de l'intervention}

Le but de l'intervention de groupe, tout comme celui du programme dans lequel elle s'insère, est d'améliorer la qualité de vie des personnes atteintes de MPOC et de leurs proches. Pour atteindre l'objectif général, qui est d'outiller ces personnes pour faire face à leur maladie et à ses impacts, l'intervention poursuit six objectifs particuliers :

- favoriser l'acceptation de la maladie;

- briser l'isolement;

- déterminer les difficultés relatives à la vie personnelle, familiale et sociale;

- reconnaître les difficultés liées à la réintégration sociale ;

- mettre en place des ressources individuelles et de groupe. 


\section{Les appuis théoriques à l'intervention}

Ce programme de groupe de soutien et d'éducation a été élaboré à partir des résultats des travaux de Lazarus et Folkman (1984) sur l'adaptation à un nouveau mode de vie et de ceux de Shulman (1984) sur le développement de l'aide mutuelle. La participation à un tel groupe (soutien et éducation) permet aux membres de bénéficier à la fois des connaissances professionnelles des intervenants et de la connaissance expérientielle des membres du groupe (Hopmeyer, 1990).

Plusieurs auteurs (Graydon et Ross, 1995 ; Lee et al., 1991) se sont déjà appuyés sur les travaux de Lazarus et Folkman (1984) pour élaborer des approches favorisant une meilleure qualité de vie des personnes atteintes de MPOC. En effet, au regard de l'adaptation à un nouveau mode de vie associé au fonctionnement des personnes atteintes de maladies pulmonaires chroniques, Lazarus et Folkman (1984) ont relevé des facteurs négatifs, tels l'anxiété et l'isolement, et un facteur positif, le soutien social.

Si les travaux de Lazarus et Folkman ont orienté le choix de thèmes à privilégier pour le contenu des rencontres, le référent théorique auquel est principalement relié le processus d'intervention de groupe est celui de l'aide mutuelle (Shulman, 1986). En ce sens, les éléments les plus souvent associés au développement de l'aide mutuelle, sont :

- le partage d'information;

- la discussion de sujets tabous;

- le sentiment d'«être dans le même bateau»;

- le soutien émotionnel.

Pour évaluer les besoins des participants nous avons procédé de deux façons. D'une part, nous avons consulté la littérature sur la MPOC et, d'autre part, les personnes ont été invitées à exprimer leurs besoins au cours de rencontres pré-groupe. Ces rencontres, en plus de permettre de vérifier les besoins et les attentes des membres, ont permis de démystifier l'intervention de groupe souvent associée, à tort, à une thérapie de groupe.

Ces besoins ${ }^{1}$ des personnes atteintes de MPOC et de leurs proches témoignent de la pertinence d'utiliser le groupe comme véhicule d'intervention et l'aide mutuelle, comme moteur à l'intervention. En effet, le groupe constitue pour ses membres un

1. Ces besoins sont : être mieux informés sur la maladie et ses effets (sur le plan social, familial, conjugal), diminuer l'isolement, améliorer le soutien social). 
milieu privilégié pour acquérir de nouvelles connaissances auprès de personnes qui sont sensibles aux problèmes et à la situation de chacune d'elles. On peut dire, à juste titre, que le développement de l'aide mutuelle entre les membres souscrit au «développement de la personne dans ses capacités individuelles, dans l'amélioration de ses relations et de son fonctionnement social» (Murphy, cité dans Paré, 1971, p. 24) et, par corollaire, contribue à améliorer la qualité de vie des personnes atteintes de MPOC.

\section{Description de l'intervention}

L'intervention se réalise dans le cadre de rencontres de groupe qui se déroulent sur une période de six semaines, à raison d'une fois par semaine. Chacune de ces rencontres, d'une durée de deux heures, traite d'un thème déterminé principalement en fonction des besoins des personnes et des appuis théoriques mentionnés précédemment. Les thèmes de chacune des rencontres sont :

- les impacts de la MPOC sur le plan psychosocial ;

- les étapes d'adaptation à la maladie et le réseau de soutien ;

- l'expression des émotions et la gestion du stress;

- la relaxation;

- la sexualité et la maladie ;

- le bilan des ateliers ou des rencontres.

Le déroulement des rencontres est le suivant: chacune débute par un court exposé, de l'intervenant principal ou des personnes-ressources, sur la thématique retenue. Cette partie rejoint l'objectif d'éducation de la démarche d'intervention. La plus grande partie du temps est consacrée à des échanges en lien avec le thème de la rencontre sur le vécu des membres du groupe et sur les moyens qu'ils expérimentent pour faire face à leur situation. Cette partie de l'atelier répond au besoin de soutien des participants. Ainsi, le partage du vécu et des expériences de vie associées à la maladie favorise le développement de l'entraide entre les participants.

\section{ÉVALUATION DE L'INTERVENTION}

L'intervention de groupe auprès des personnes atteintes de MPOC a été peu évaluée jusqu'à maintenant. Les évaluations réalisées ont porté principalement sur l'aspect éducatif de la 
démarche et les groupes évalués étaient composés de personnes atteintes d'asthme et non de maladie pulmonaire obstructive chronique (Thapar, 1994 ; Fox, 1989 ; Sheldon et Monk, 1990).

Désireux de mieux connaître l'impact de l'intervention de groupe sur les participants, nous avons, dans un premier temps, procédé à une évaluation pour vérifier si l'intervention produit des modifications de comportements chez les personnes atteintes de MPOC et chez leurs proches. Dans un deuxième temps, nous avons évalué le développement de l'aide mutuelle sous l'angle des quatre éléments ${ }^{2}$ les plus souvent identifiés à son développement et contribuant à l'amélioration de la qualité de vie des participants.

\section{Devis d'évaluation}

Le devis d'évaluation retenu pour juger des effets de l'intervention chez les participants au groupe est de type quasi expérimental sans groupe de contrôle. Dans le contexte de cette intervention, la constitution d'un groupe de contrôle était impossible, car les personnes atteintes de MPOC inscrites dans le programme de réadaptation participaient toutes à l'intervention de groupe. Pour des raisons éthiques, il nous a semblé inapproprié de reporter, à des fins d'évaluation, la participation d'un certain nombre de personnes à une session ultérieure.

Notre groupe expérimental était composé de douze personnes ayant participé à l'intervention qui s'est déroulée à l'automne 1995. Les sujets de ce groupe ont répondu à un questionnaire à deux reprises (pré et post-intervention).

Il nous apparaît important dès maintenant de signaler les limites que représentent le petit nombre de sujets composant le groupe expérimental et l'impossibilité de constituer un groupe de contrôle. L'interprétation des résultats de cette étude devra en tenir compte. Conscients de ces limites, nous croyons que les résultats de cette étude pourront malgré tout apporter un éclairage pertinent sur la question à l'étude. Une recherche ultérieure pourra peut-être se réaliser dans des conditions permettant un contrôle de ces limites.

2. Ces éléments sont le partage d'information, la discussion de sujets tabous, le sentiment $\mathrm{d}^{\prime}$ " être dans le même bateau», le soutien émotionnel. 


\section{Cadre d'analyse}

Pour répondre au but visé par l'évaluation qui, rappelons-le, était de mesurer les résultats de l'intervention de groupe, nous avons identifié huit dimensions de la qualité de vie des personnes qui font face à la maladie et à ses conséquences. Chacune de ces dimensions a été mesurée à partir d'une série d'indicateurs (voir tableau 1). Chacun des indicateurs a été opérationnalisé au moyen de vignettes proposées aux répondants à l'intérieur d'un questionnaire. Chacune de ces vignettes (voir tableau 2) renvoie à un comportement attendu de la part du sujet à la suite de l'intervention. Les dimensions et leurs indicateurs ont été identifiés à partir d'une recension des écrits portant sur le vécu des personnes atteintes de MPOC et d'observations cliniques.

Toutes les dimensions interagissent pour favoriser chez les participants du groupe atteints d'une maladie pulmonaire obstructive une meilleure qualité de vie. Ainsi, pour les participants, le groupe constitue d'abord un lieu favorisant l'identification et la verbalisation des difficultés reliées à la maladie, que ce soit sur le plan personnel, familial ou social. Ce faisant, le groupe amène les participants à exprimer les sentiments et émotions qu'ils ressentent et qui sont liés aux impacts de la maladie dans leur vie. Comme les participants ont plusieurs occasions de partager les frustrations vécues en raison des limites inhérentes à leur maladie, cette démarche constitue un exutoire qui peut contribuer à un début d'acceptation de leur maladie. Plus le processus d'acceptation progresse, plus le participant est capable d'envisager l'adoption d'un style de vie conforme à ses capacités. Pour y parvenir, il devra identifier ses capacités résiduelles et en tenir compte dans son quotidien. L'adoption d'un style de vie implique aussi de mieux gérer les différents stress de la vie quotidienne, en sachant mieux comment y réagir. Dans ce processus d'apprentissage le participant apprend à mieux se connaître, à mieux identifier ses capacités, à trouver des solutions réalistes eu égard à sa situation et à élaborer des stratégies inspirées des échanges avec les autres membres du groupe.

\section{Instruments de mesure}

Les instruments utilisés pour mesurer les effets de l'intervention et le processus de groupe sont contenus à l'intérieur d'un questionnaire élaboré à partir des observations cliniques de travailleurs sociaux. Le questionnaire a été soumis à une procédure de prétest 
avant d'être rédigé dans sa forme définitive. Ce questionnaire a été administré avant et après intervention. Il est constitué principalement de questions fermées et de quelques questions ouvertes.

\section{Tableau 1}

Dimensions et indicateurs de la qualité de vie des personnes atteintes de MPOC

\section{Acceptation}

de la maladie

Prendre conscience de ses limites

Respecter ses limites

Prendre conscience de l'importance de se donner un rythme

Vivre au jour le jour

Réagir avec ouverture à sa maladie

Gestion du stress

Prendre conscience du stress

Réagir au stress

Identification et verbalisation

des difficultés

Nommer les principales difficultés

Identifier la difficulté

la plus importante

Reconnaître l'importance

des difficultés identifiées
Adoption d'un style de vie conforme à sa situation

Identifier l'action en fonction de ses limites

Agir conformément à ses limites

Déterminer un rythme conforme à ses capacités

S'ajuster au quotidien

Parler facilement de sa maladie

Rester actif

\section{Maximisation des capacités} résiduelles

Participer à différentes activités sociales, domestiques et autres

\section{Émergence de solutions}

Solliciter l'aide et le soutien de la famille

Solliciter l'aide et le soutien de l'entourage

\section{Bris de l'isolement}

Favoriser les contacts avec les amis

Favoriser les contacts avec la famille

\section{Expression des sentiments de colère}

Manifester de la colère à l'égard des membres de la famille

Manifester de la colère à l'égard de l'entourage 
TABLEAU 2

Exemples de vignettes

\begin{tabular}{|c|c|c|}
\hline Dimension & Indicateur & Vignette \\
\hline $\begin{array}{l}\text { Acceptation } \\
\text { de la maladie }\end{array}$ & $\begin{array}{l}\text { Connaissance } \\
\text { de son rythme } \\
\text { en fonction } \\
\text { de ses capacités }\end{array}$ & $\begin{array}{l}\text { La dernière fois que vous avez } \\
\text { transporté vos sacs d'épicerie } \\
\text { sur une certaine distance, vous vous } \\
\text { êtes senti essoufflé avant d'arriver. } \\
\text { Comment avez-vous réagi } \\
\text { à ce moment-là? } \\
\text { - Vous vous êtes arrêté en vous disant } \\
\text { que vous ne transporteriez plus } \\
\text { jamais de sacs d'épicerie. } \\
\text { - Vous avez décidé de vous rendre } \\
\text { à destination malgré votre } \\
\text { essoufflement. } \\
\text { - Vous êtes resté figé en ne sachant pas } \\
\text { quoi faire avec vos sacs. } \\
\text { - Vous vous êtes arrêté pour faire une } \\
\text { pause avant de continuer. } \\
\text { - Autres possibilités (précisez). }\end{array}$ \\
\hline $\begin{array}{l}\text { Gestion } \\
\text { du stress }\end{array}$ & $\begin{array}{l}\text { Réaction } \\
\text { au stress }\end{array}$ & $\begin{array}{l}\text { Vous apprenez qu'un de vos amis } \\
\text { souffre de cancer. Qu'est-ce que } \\
\text { cela vous fait? } \\
\text { - Vous préférez ne pas voir cet ami. } \\
\text { - Vous décidez d'aller le voir même } \\
\text { - si cela vous affecte beaucoup. } \\
\text { - Vous ne savez pas quoi faire. } \\
\text { - Vous irez le voir quand vous vous } \\
\text { - } \text { sentirez capable de le faire. } \\
\text { - Autres possibilités (précisez). }\end{array}$ \\
\hline
\end{tabular}

Les questions fermées ont été formulées sous forme de vignettes illustrant des situations de la vie réelle auxquelles font face quotidiennement les personnes atteintes de MPOC. Une attention particulière a été portée à la formulation de ces situations, afin que celles-ci traduisent le plus fidèlement possible le vécu décrit par les personnes atteintes de MPOC aux différents intervenants qui sont en relation avec elles. 
Dans le questionnaire administré au début de l'intervention, des informations ont été recueillies sur les caractéristiques des participants (état civil, présence d'enfants, état de santé, participation à des activités de groupe ) et sur la nature de leur participation aux activités. Dans le questionnaire administré après intervention, des opinions ont été recueillies sur la pertinence des thèmes abordés au cours des rencontres et les changements souhaités dans le déroulement de celles-ci. La contribution de la dynamique de groupe a également évaluée par une série d'énoncés ${ }^{3}$ correspondant aux effets attendus de l'intervention de groupe (Home et Darveau-Fournier, 1980; Shulman, 1986; Gitterman, 1989 ; Shapiro, 1977).

Informations recueillies à partir des questionnaires

\section{Avant intervention}

- Difficultés quotidiennes à partir de vignettes comportant des choix de réponses multiples

- Informations sociodémographiques

\section{Après intervention}

- Difficultés quotidiennes à partir de vignettes comportant des choix de réponses multiples (les mêmes questions posées avant intervention)

- Questions de satisfaction sur le contenu des rencontres

- Questions d'évaluation de l'aide mutuelle

Le protocole comprenait donc deux collectes de données effectuées à des moments différents du processus d'évaluation. Les douze personnes qui ont participé aux sessions de l'automne 1995 ont été rencontrées en groupe avant le début de l'intervention et à la fin de celle-ci afin de répondre au questionnaire.

\section{Population à l'étude}

Les deux tiers des participants à l'intervention sont des hommes (8 sur 12 ). De même, les deux tiers des participants vivent avec un conjoint ou une conjointe (8 sur 12). Dix participants sur

3. Ces énoncés sont les suivants : constat que d'autres personnes vivent des difficultés semblables, partage de vécu commun, recherche de solutions, sentiment d'espoir, stimulation par les témoignages et les actions des autres personnes, soutien des membres du groupe, application des connaissances transmises, nouveaux apprentissages. 
douze ont des enfants et, pour huit d'entre eux, les enfants ne demeurent pas au domicile des parents. Nous nous sommes aussi intéressés à la proximité géographique des enfants des huit sujets qui ont répondu que leurs enfants n'habitaient pas avec eux. Six d'entre eux ont dit que leurs enfants demeuraient à moins de $10 \mathrm{~km}$ de leur domicile. La presque totalité des participants (11 sur 12) ont plus de 65 ans.

Les résultats de l'intervention seront d'abord évalués en tenant compte des changements observés dans les comportements des participants au cours de la période s'écoulant entre le début et la fin de l'intervention. Sera ensuite analysé le processus d'aide mutuelle tel qu'évalué par les participants à la fin de l'intervention.

\section{Changements de comportements chez les répondants}

Les effets attendus de l'intervention auprès des participants ont été évalués par rapport à huit dimensions, dont sept ont été mesurées à partir d'indicateurs quantitatifs. La mesure des

TABLEAU 3

Scores des dimensions pour l'ensemble des participants au groupe avant et après l'intervention et variation des scores

\begin{tabular}{|c|c|c|c|c|}
\hline Dimensions & Avant & Après & Variation & $\mathbf{N}$ \\
\hline - Acceptation de la maladie & 73,24 & 75,00 & $+1,76$ & 11 \\
\hline $\begin{array}{l}\text { Adoption d'un style de vie } \\
\text { conforme à sa situation }\end{array}$ & 75,50 & 78,90 & $+3,40$ & 12 \\
\hline - Gestion du stress & 73,33 & 75,00 & $+1,67$ & 10 \\
\hline - Bris de l'isolement & 86,11 & 86,11 & 0,00 & 12 \\
\hline $\begin{array}{l}\text { - Maximisation des capacités } \\
\text { résiduelles }\end{array}$ & 59,50 & 64,88 & $+5,38$ & 12 \\
\hline - Émergence de solutions & 43,89 & 43,40 & $-0,40$ & 12 \\
\hline $\begin{array}{l}\text { - Expression des sentiments } \\
\text { de colère }\end{array}$ & 38,89 & 40,27 & $+1,38$ & 12 \\
\hline
\end{tabular}


dimensions quantitatives ${ }^{4}$ et de leurs indicateurs a été obtenue à partir d'échelles ordinales qui ont été par la suite transformées en pourcentages de façon à standardiser les résultats et ainsi faciliter les comparaisons.

L'analyse des sept dimensions mesurées sur la base d'indicateurs quantitatifs (voir tableau 3) révèle une amélioration des comportements sur toutes les dimensions à l'exclusion de deux - bris de l'isolement et émergence de solutions -, pour lesquelles le score demeure stable.

L'analyse des variations des valeurs standardisées de chacun des indicateurs permet de constater que les comportements qui changent le plus avant et après intervention sont associés à quatre des cinq dimensions présentant des améliorations, soit acceptation de la maladie, adoption d'un style de vie conforme à sa situation, expression des sentiments de colère et maximisation des capacités résiduelles (voir tableau 4). Six comportements parmi les vingt qui composent les sept dimensions affichent des changements d'importance moyenne ${ }^{5}$ au cours de la période avant / après intervention et trois autres changent de façon plus marquée.

Pour trois des cinq indicateurs de la dimension acceptation de la maladie on observe des changements d'ordre moyen, alors que pour les deux autres, «Prendre conscience de ses limites» et "Prendre conscience de l'importance de se donner un rythme», il y a absence de changements. Le comportement qui affiche la plus forte amélioration est «Réagir avec ouverture à sa maladie».

Tous les indicateurs de la dimension adoption d'un style de vie conforme à sa situation présentent des changements positifs dont les plus importants sont «Identifier l'action en fonction de ses limites» et «Déterminer un rythme conforme à ses capacités».

Pour la dimension gestion du stress, mesurée à partir de deux indicateurs, on constate que l'indicateur «Prise de conscience du stress » demeure stable, alors que celui de «Réaction au stress » s'améliore légèrement.

4. Une des huit dimensions de la qualité de vie, identification et verbalisation des difficultés, a été évaluée qualitativement et sera traitée plus loin dans cette section.

5. Nous considérons les variations se situant entre 5 et 8 points comme des changements «moyens» et les variations de plus de 8 points comme des changements "plus importants ». 
Tableau 4

Valeurs des indicateurs pour I'ensemble des participants au groupe avant et après l'intervention et variation des scores

\begin{tabular}{lllll}
\hline & Avant & Après & Variation & N \\
\hline Acceptation de la maladie & & & & \\
- Prendre conscience de ses limites & 66,70 & 63,90 & $-2,80$ & 11 \\
- Respecter ses limites & 79,15 & 87,50 & $+8,35$ & 12 \\
- Prendre conscience de l'impor- & 83,35 & 75,00 & $\underline{-8,35}$ & 12 \\
$\quad$ tance de se donner un rythme & & & & \\
- Vivre au jour le jour & 59,10 & 66,60 & $+7,50$ & 12 \\
- Réagir avec ouverture à sa maladie & 77,25 & 87,50 & $\pm 10,25$ & 12
\end{tabular}

\section{Adoption d'un style de vie conforme à sa situation}

- Identifier l'action en fonction de ses limites

- Agir conformément à ses limites

- Déterminer un rythme conforme à ses capacités

- S'ajuster au quotidien

- Parler facilement de sa maladie

- Rester actif
66,67

77,78

$+11,11$

79,17

$+4,17$

75,00

70,83

83,33

$+8,33$

$+4,17$

12

63,64

75,00

$+6,36$

$+3,79$

78,79

75,00

78,79

51,51

51,51

0,00

11

93,33

96,97

$+3,64$

10

\section{Bris de l'isolement}

- Favoriser les contacts avec les amis

- Favoriser les contacts avec la famille 80,56

91,67

$0,00 \quad 12$

80,56

0,00

12

\section{Émergence de solutions}

- Solliciter l'aide et le soutien des membres de la famille

$\begin{array}{llll}48,61 & 43,75 & -4,86 & 12\end{array}$

- Solliciter l'aide et le soutien

48,61

43,75

$-4,86$ de l'entourage

\section{Expression des sentiments de colère}

- Manifester de la colère vis-à-vis des membres de la famille

$36,11 \quad 41,66+5,50 \quad 12$

- Manifester de la colère vis-à-vis

$\begin{array}{lll}41,66 & 38,89 & -2,77\end{array}$ des personnes de l'entourage

\section{Maximisation des capacités résiduelles}

- Participer à différentes activités 
En ce qui concerne la dimension expression des sentiments de colère, un seul des deux indicateurs évalués présente un changement positif moyen, l'autre affichant un changement négatif. Ainsi, après l'intervention, les sujets manifestent moins de colère vis-à-vis de la famille, alors qu'à l'égard de l'entourage ils en manifestent davantage.

À la dimension maximisation des capacités résiduelles, l'indicateur mesuré, «Implication dans différentes activités», présente un changement positif moyen.

En ce qui concerne l'analyse de la dimension bris de l'isolement, aucun changement n'a été observé pour les deux indicateurs évalués. Les sujets n'ont pas plus de contact avec les membres de la famille et de l'entourage avant qu'après l'intervention. Enfin, pour ce qui est de la dimension émergence de solutions, les deux indicateurs évalués présentent des changements négatifs. Ainsi, les participants sollicitent moins l'aide et le soutien des membres de la famille et de l'entourage avant qu'après l'intervention.

Un autre aspect de la qualité de vie des personnes atteintes de MPOC a aussi été évalué; il s'agit de l'identification et de la verbalisation des difficultés. Pour évaluer cet aspect, nous avons demandé aux sujets :

- de nommer les difficultés associées à leur maladie qu'ils ont rencontrées avant la période d'intervention;

- de nommer les difficultés associées à leur maladie qu'ils ont vécues pendant l'intervention;

- d'identifier des aspects de leur vie personnelle, familiale ou sociale qu'ils souhaitaient améliorer pour s'assurer une meilleure qualité de vie.

Toutes les questions concernant ces aspects étaient de type «ouvert». Nous en rapporterons ici des résultats fondés sur l'analyse du contenu des énoncés.

L'ensemble des répondants a été en mesure d'identifier des difficultés éprouvées dans les mois précédant le début de l'intervention. Cependant, quant aux aspects de leur vie qu'ils espèrent pouvoir changer dans les deux mois suivant l'intervention, cinq des douze participants n'ont rien mentionné.

$\mathrm{Au}$ cours des semaines où s'est déroulée l'intervention, cinq sujets n'ont signalé aucune difficulté. Enfin, les six participants qui ont indiqué clairement des aspects de leur vie personnelle, familiale et sociale qu'ils souhaitaient voir améliorer ont aussi 
clairement signalé des changements qui se sont réalisés au cours des deux derniers mois précédant la fin de l'intervention.

\section{Le processus de groupe}

Pour évaluer le processus de groupe, les participants ont été invités à se prononcer sur huit énoncés décrivant des aspects caractéristiques du processus d'aide mutuelle (Shulman, 1984). Chacun des énoncés dont le contenu est présenté à la figure 1 était structuré sous la forme d'une échelle de type Likert en 4 points (Tout à fait d'accord, D'accord, En désaccord, Tout à fait en désaccord).

Figure 1

Degré d'adhésion à des énoncés relatifs aux contributions de l'intervention de groupe

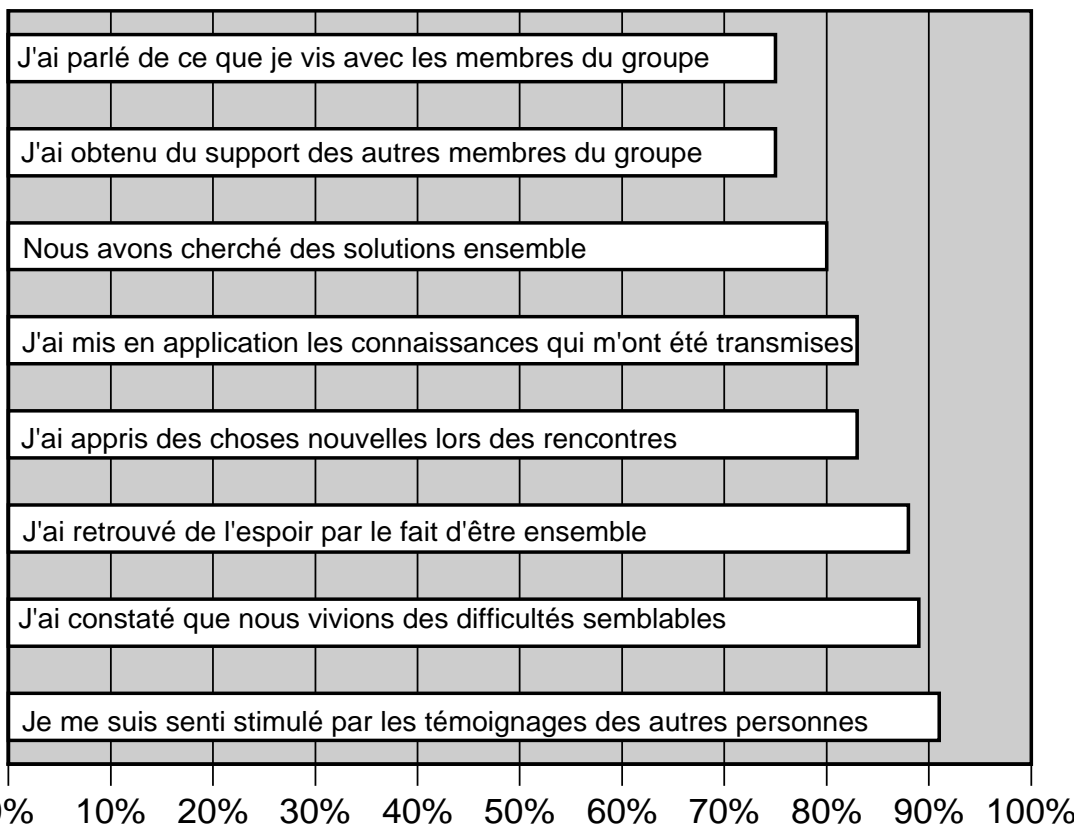

Le score associé à chacun des énoncés est ici exprimé sur un continuum de base $0-100$, où 0 exprime un désaccord total et 100 un accord total. Il s'agit toujours d'une évaluation globale de l'ensemble des sujets. 
Comme le démontre la figure 1 , le niveau d'accord ne descend jamais sous la barre des $75 \%$.

C'est à l'égard de la stimulation par les témoignages des autres que les participants manifestent le plus haut niveau d'accord. Les énoncés à l'égard desquels on observe le plus faible niveau $\mathrm{d}$ 'accord est parler de ce que je vis avec les autres (dire aux autres) et obtenir du soutien des autres membres du groupe.

$\mathrm{Si}$, d'un côté, on observe un niveau passablement semblable d'un énoncé à l'autre, de l'autre il est intéressant de signaler la différence entre les deux énoncés qui se situent aux deux extrémités du continuum. Il semble plus difficile de manifester son accord vis-à-vis de la contribution de l'intervention lorsqu'elle est associée à ce qu'on partage avec le groupe et à ce qu'on obtient comme soutien des membres, contrairement à ce qu'on retire de ce qui est exprimé par les autres.

\section{DISCUSSION}

En ce qui regarde l'objectif principal de l'intervention de groupe, qui est d'outiller les participants pour faire face à la situation associée à leur maladie, nous avons vérifié si l'intervention contribue à provoquer les changements attendus, c'est-à-dire une meilleure acceptation de la maladie, l'adoption d'un style de vie conforme à la situation, l'utilisation de capacités résiduelles, l'émergence de solutions, la gestion des réactions de stress et des sentiments de colère, la diminution de l'isolement.

La dimension acceptation de la maladie est une mesure du contrôle «cognitif» que le sujet a sur sa maladie, alors que la dimension adoption d'un style de vie conforme à sa situation est un estimateur du contrôle "comportemental». Les résultats de l'évaluation permettent de constater que l'intervention de groupe joue un rôle significatif sur ces deux aspects. Ainsi, il est possible de soutenir qu'en ce qui regarde le contrôle "cognitif» de leur maladie les participants à l'intervention de groupe comprennent davantage qu'il est important de respecter ses limites, de vivre au jour le jour et de réagir avec ouverture à sa maladie. Cependant, les participants sont moins conscients de l'importance de se donner un rythme. Cette situation pourrait vraisemblablement s'expliquer par le fait que, pour se protéger d'une situation difficile à accepter, certaines personnes atteintes de MPOC développent des mécanismes de défense (Rabinowitz et Florian, 1992). Le mécanisme de défense qui consiste à ne pas 
modifier son rythme de vie, même si la condition physiologique de la personne atteinte se détériore, correspond à ce que Agle et Baum (1977) qualifient de répression ou suppression.

En matière de contrôle "comportemental», les participants à l'intervention de groupe accordent une plus grande priorité à l'action. Cela se vérifie au moyen de plusieurs indicateurs: par l'identification des actions en fonction de leurs limites, par le fait de déterminer un rythme selon leurs capacités (ce qui est l'inverse de ce qu'on observait plus haut pour la composante cognitive de l'évaluation) et par la facilité à parler de leur maladie.

L'analyse des résultats de la dimension maximisation des capacités résiduelles nous amène à conclure que les actions posées par les participants vis-à-vis des capacités qu'ils possèdent toujours les amènent à s'engager davantage dans différentes activités sociales, domestiques et autres. Ce comportement s'ajoute alors à ceux déjà associés à l'action et confirme encore une fois le rôle potentiellement joué par l'intervention de groupe pour modifier de façon positive l'implication des participants dans différentes sphères de leur vie. Cette modification de comportement après intervention nous permet donc d'associer positivement l'intervention et la plus grande participation des membres à différentes activités. De façon générale, en effet, les auteurs soulignent la diminution des activités sociales chez les personnes atteintes de MPOC ainsi que chez leurs proches (Rabinowitz et Florian, 1992; Beck et al., 1988; Sexton et Munro, 1988), ce qui n'est pas le cas chez les participants à l'intervention de groupe.

L'analyse de la dimension expression des sentiments de colère démontre que depuis leur participation à l'intervention de groupe les participants expriment moins de colère au sein de leur milieu familial.

Les résultats obtenus pour la dimension bris de l'isolement sont très différents de ceux rapportés par Gayle et al. (1993), qui observent une situation d'isolement chez les personnes atteintes de MPOC ainsi que chez leurs proches. Il est important de préciser que notre étude est de type quantitative et que nous avons évalué la fréquence des contacts des participants avec leur famille et leur entourage et non pas la qualité de ceux-ci. Pour De Jong-Gierveld (1987), ce qui influence davantage la qualité de vie des personnes atteintes de MPOC, ce n'est pas le nombre mais la qualité des contacts, ce que notre étude ne nous a pas permis d'évaluer.

En ce qui regarde la capacité des participants à utiliser le soutien des membres de la famille ou de l'entourage (émergence 
de solutions), on note que les changements ne vont pas dans le sens attendu. Pour expliquer cette situation, nous posons l'hypothèse que les participants au groupe devaient avoir moins besoin d'utiliser le soutien de la famille et de l'entourage, parce que, d'une part, l'intervention de groupe peut avoir contribué de façon significative à l'adoption d'un style de vie conforme à sa situation et à la maximisation des capacités résiduelles. D'autre part, leur participation au programme de réadaptation a pu également contribuer au fait que les participants ressentent moins le besoin d'utiliser le soutien des membres de la famille ou de l'entourage. Une étude qualitative permettrait de vérifier ces hypothèses.

Les participants à l'intervention présentent une variation positive par rapport à la dimension réaction au stress. Connaissant le niveau d'anxiété vécu par les personnes atteintes de MPOC (Beck et al. 1988; Kinsman et al., 1983; Dudley et al., 1980 ; Rabinowitz et Florian, 1992), il est important de souligner que même une variation que l'on pourrait qualifier de non significative peut améliorer la qualité de vie de ces personnes. Pour Lazarus et Folkman (1984), la réaction au stress est d'autant plus importante pour les personnes atteintes de MPOC qu'elle affecte le fonctionnement de la vie quotidienne de celles-ci.

En ce qui concerne l'apport du groupe les résultats de l'évaluation de la part des sujets témoignent de l'expression claire d'un apport positif de celui-ci sur les aspects visés par les énoncés qui leur ont été proposés. Cette évaluation supporte l'importance pour les participants de ce type d'intervention dans le programme de réadaptation. Au-delà de l'aspect éducatif d'une partie de l'intervention, les personnes atteintes de MPOC ont manifesté une grande satisfaction à se rencontrer lors des rencontres, à partager leur vécu commun, à échanger sur les moyens utilisés par chacun pour améliorer sa qualité de vie. Même s'il est parfois difficile de parler des difficultés associées à la maladie, les témoignages des participants demeurent des sources de stimulation et contribuent à développer et à maintenir un sentiment d'espoir.

Quoique les changements observés ne soient pas très marqués en ordre de grandeur, il est important de considérer la très courte période écoulée entre les deux mesures. Dans ce contexte, ces différences devraient être considérées comme indicatives de changements effectifs qui sont en accord avec les résultats attendus de l'intervention. 


\section{CONCLUSION}

L'intervention de groupe axée sur le soutien et l'éducation représente, comme le révèlent les résultats de l'évaluation, une stratégie efficace pour venir en aide aux personnes atteintes de MPOC et à leurs proches. Toutefois, comme toute démarche se situe dans une dynamique systémique, on ne peut attribuer tous les résultats à une seule intervention. L'évaluation effectuée a néanmoins été élaborée de telle sorte qu'on puisse saisir les effets les plus étroitement associés à l'intervention de groupe.

Ainsi, les constats résultant de l'analyse nous amènent à conclure à certains effets de l'intervention de groupe. D'abord, l'intervention de groupe favoriserait chez les participants l'amorce d'une prise de conscience de la maladie et de ses impacts sur leur vie personnelle, familiale et sociale. Dans un deuxième temps, l'intervention de groupe contribuerait à outiller les participants pour faire face à leur situation puisque, après l'intervention, on observe des changements de comportements pour les indicateurs qui sont associés à l'action, soit: maximiser ses capacités résiduelles, adapter sa vie à sa situation, réagir positivement au stress, éprouver moins de colère envers la famille.

En particulier, nous pouvons croire que l'intervention de groupe atteint l'objectif spécifique poursuivi par la démarche, qui est d'amener la personne à développer des moyens permettant d'apprendre à vivre avec cette maladie et ses effets. Le travail effectué dans le cadre de l'intervention de groupe permet vraisemblablement aux participants de prendre conscience des capacités et des moyens utiles pour affronter leur situation. L'intervention de groupe réalisée auprès des personnes atteintes de MPOC contribue probablement aussi de façon significative à augmenter le contrôle que celles-ci ont sur leur propre vie.

La revue de littérature effectuée dans le cadre de cette recherche ne nous a pas permis d'identifier de recherches de type évaluatif portant sur une intervention de groupe (soutien et éducation) comme celle menée à l'hôpital Laval. Toutefois, en ce qui concerne l'aspect éducatif de la démarche d'intervention de groupe, nos constats vont dans le même sens que ceux de Thapar (1994), c'est-à-dire que l'intervention de groupe contribue à une meilleure connaissance de la maladie et de ses effets.

Enfin, on ne pouvait certainement pas s'attendre à observer des changements radicaux de comportements au cours de la courte période entourant l'intervention de groupe. En effet, 
changer un comportement prend du temps. Toutefois, les changements observés relativement à certains comportements vont tous dans le même sens: dans l'ensemble, les participants au groupe exercent un meilleur contrôle sur leur vie personnelle, familiale et sociale, ce qui contribue à améliorer leur qualité de vie.

À la lumière des résultats de l'évaluation, il est intéressant de noter à quel point l'intervention de groupe a pu contribuer à inciter les participants à poser des gestes concrets visant l'amélioration de leur situation. La priorité à l'action nous a semblé être un aspect déterminant dans l'amorce d'un changement vers une meilleure qualité de vie. Cette constatation nous a amenés à revoir le contenu de l'intervention pour y ajouter une dimension nouvelle, qui sera centrée sur l'importance de l'action dans l'amélioration d'une situation de vie difficile.

Précisons en terminant, comme nous l'avons déjà mentionné, que les résultats de notre recherche comportent quelques limites. Toutefois, les conclusions de notre démarche devraient aider la poursuite d'autres recherches de même nature, de façon à confirmer et à renforcer la vraisemblance des résultats de la présente étude.

\section{Références bibliographiques}

AGLE, D., et G. BAUM (1977). "Psychological aspects of chronic obstructive lung disease", Medical Clinics of North America, vol. 61, p. 749-758.

Beck, J.G., S.K. Scott, R.B. Teague, F.I. Perez et G.A. Brown (1988). «Correlates of daily impairment in COPD », Rehabilitation Psycho$\log y$, vol. 33, p. 77-87.

BRACUK, D. (1993). Faire face à la MPOC. Traiter et vivre avec la maladie pulmonaire obstructive chronique, Association médicale canadienne: Éditions Grosvenor, 100 p.

CELle, B.R. (1995). "Pulmonary Rehabilitation in Patients with COPD », Am Respir Crit Care Med, vol. 152, p. 861-864.

Dudley, D., E. Glaser, B. Jorgensson et D. LOGAN (1980). «Psychosocial concomitants to rehabilitation in chronic obstructive pulmonary desease : part one. Psychosocial and psychological considerations ", Chest, vol. 77, n ${ }^{\circ} 3$, p. 413-420.

FoX, D. (1989). "Effect of small group education on the outcome of chronic asthma », Journal of the Royal College of General Practitioners, vol. 39, p. 182-186. 
GAYle, K.C., M.J. Foxall et C.R. BARron (1993). "Loneliness, Depression, and Social Support of Patients with COPD and Their Spouses », Public Health Nursing, vol. 10, $\mathrm{n}^{\circ} 4$, p. 245-251.

GIERVELD, L. DE JONG (1987). «Developing and testing a model of loneliness », Journal of Personality and Social Psychology, vol. 53, p. 119-128.

GitTERMAN, A. (1989). "Building mutual support in groups », Social Work with Groups, vol. 12, n ${ }^{\circ} 2$, p. 5-21.

GRAYDON, J.E., et E. ROSS (1995). «Influence of Symptoms, Lung Function, Mood, and Social Support on Level of Functioning of Patients with COPD», Research in Nursing \& Health, vol. 18, p. 525-533.

Home, A., et L. DARveau-Fournier (1980). «La spécificité du service social des groupes ", Service social, vol. 37, no 1-2, p. 16-31.

HOPMEYER, E. (1990). «Entraide et service social des groupes », Service social, vol. $39, \mathrm{n}^{\circ} 1$, p. 64-74.

KINSMAN, R., et al. (1983). "Symptoms and experiences in chronic bronchitis and emphysema », Chest, vol. 83, n ${ }^{\circ} 2$, p. 755-761.

LAUZON, S., et E. ADAM (1996). La personne âgée et ses besoins, Montréal: Éditions du Renouveau pédagogique, $867 \mathrm{p}$.

LAZARUS, R.S., et S. FOLKMAN (1984). Stress, appraisal and coping, New York: Springer.

LEE, R.N.F., J.E. GRAYDON et E. ROSS (1991). «Effects of psychological well-being, physical status, and social support on oxygen-dependant COPD patients' level of functioning », Research in Nursing \& Health, vol. 14, p. 323-328.

Light, R.W., et al. (1985). "Prevalence of Depression and Anxiety in Patients with COPD», Chest, vol. 87, p. 35-38.

MANFREDA, Y.M. et W. LITVIN (1992). «Morbidity and mortality from chronic obstructive pulmonary desease », American Review Respiratory Disease, vol. 140, p. S19-S26.

Ojanen, M., A. LAhdensuo, J. LAitinen et J.Karvonen (1993). «Psychosocial Changes in Patients Participating in a Chronic Obstructive Pulmonary Disease Rehabilitation Program», Respiration, vol. 60, p. 96-102.

PARÉ, S. (1971). Groupes et service social, Québec: Presses de l'Université Laval, p. 9-23.

RABINOWITZ, S., et V. Florian (1992). "Chronic Obstructive Pulmonary Disease: Psycho-Social Issues and Treatment Goals», Social Work in Health Care, vol. 16, $\mathrm{n}^{\circ} 4$, p. 69-86.

Ries, A.L., R.M. KAPlaN, T.M. Limberg et L.M. Prewitt (1995). " Effects of Pulmonary Rehabilitation on Physiologic and Psychosocial 